\title{
Medizinische Dissertation: Wie unterscheiden sich erfolgreiche von abgebrochenen Forschungsprojekten?
}

M. Dewey

\author{
Medical dissertation: differences between successful and \\ aborted research projects
}

Hintergrund und Fragestellung: Es gibt bis heute keine Untersuchungen an einer medizinischen Fakultät zum Vergleich zwischen erfolgreich und erfolglos verlaufenden Promotionsvorhaben. Die Feststellung von Unterschieden zwischen erfolgreichen und erfolglosen Dissertationen war deshalb Ziel dieser Studie.

Probanden und Methodik: Es wurden alle Medizinstudenten des 11. bis 14. Fachsemesters der Charité $(n=1036$; 560 Frauen [54,1\%] und 476 Männer [45,9\%]) per Fragebogen zu Ihrer Promotionssituation befragt. Beschrieben werden konnte das momentane und bis zu zwei abgebrochene Promotionsvorhaben. Die eingehenden Bögen wurden in „erfolgreiche“ und „abgebrochene" Promotionen klassifiziert.

Ergebnisse: Insgesamt beantworteten 324 Studenten den Fragebogen zur Promotionssituation und beschrieben 348 Promotionsvorhaben an der Charité (Rücklaufquote 31\%). Unter diesen wurden 72 erfolgreiche und 65 abgebrochene Arbeiten identifiziert. Die erfolgreichen Arbeiten wurden signifikant besser betreut (Betreuer mit ausreichend Zeit [64\% vs. 29\%], gute Hilfe des Betreuers [57\% vs. 9\%]). Die Planung der erfolgreichen $\mathrm{Ar}$ beiten war ebenfalls signifikant überlegen (eingehaltene Zusagen [ $42 \%$ vs. $5 \%$ ], finanzielle Unterstützung [39\% vs. $8 \%]$ ). Bei den erfolgreichen Arbeiten gab es außerdem signifikant häufiger ein Bemühen um den Studenten als zukünftigen Forschungspartner (50\% vs. $17 \%$ ).

Folgerung: Erfolgreiche Promotionen werden deutlich besser betreut und vorbereitet. Um die Erfolgschancen einer Promotion zu verbessern, muss eine bessere Planung und Supervision erzielt werden. Des Weiteren sind eine finanzielle Unterstützung und eine gute Vorbereitung der Promovenden auf wissenschaftliches Arbeiten, z.B. durch spezielle Kurse, wichtige Förderungsinstrumente.
Background and objective: To obtain the title „Doctor medicinae“ in Germany a dissertation is required. There are no data currently available to compare successful and unsuccessful student research projects. This study's aim was to determine observational differences between the two groups of successful and unsuccessful dissertations pursued by medical students.

Methods: All medical students of the $11^{\text {th }}$ to $14^{\text {th }}$ terms of study at the Charité Hospital in Berlin ( $n=1036 ; 560$ females [54.1\%] and 476 males [45.9\%]) received a questionnaire, which invited them to describe the current and/or up to two aborted dissertations. The returned questionnaires were classified into „successful“ and „unsuccessful“ dissertations.

Results: 324 (31\%) students responded, describing 348 dissertation projects at the Charité. Of these, 72 were identified as successful and 65 as unsuccessful. Successful dissertations were significantly better supervised (the supervisor was available more often [64\% vs. $29 \%$ ]; the supervisor was more often helpful [57\% vs. 9\%]). Further, planning of successful projects was significantly better (dissertation faithful to agreed-on specifications [ $42 \%$ vs. $5 \%$ ]; financially supported [ $39 \%$ vs. $8 \%]$ ). In addition, efforts to attract the student as a future research partner were significantly more frequent when the dissertation was successful ( $50 \%$ vs. $17 \%$ ).

Conclusion: Successful dissertations are clearly the result of better preparation and continuous close supervision. To improve the chances of success, better planning and supervision are essential. Moreover, financial support and proper preparation for scientific work, e.g., by special courses, maybe promising instruments to increase the fraction of successful dissertations. Fachschaftsinitiative Medizin Universitätsklinikum Charité, Medizinische Fakultät der Humboldt-Universität zu Berlin 
In Deutschland wird der akademische Grad des „Dr. med.“ nur nach Anfertigung einer Promotionsarbeit verliehen. Dieser ist für die ärztliche Tätigkeit jedoch keine zwingende Voraussetzung. So sind in Berlin beispielsweise 58\% aller tätigen Ärzte promoviert (Mitteilung der EDV-Abteilung der Berliner Ärztekammer). Zumeist wird die Arbeit an der Promotion während des Studiums begonnen. In Ländern wie den USA ist der Anteil von studentischer Forschung aufgrund ihres freiwilligen Charakters geringer (7). Studentische Forschung spielt jedoch eine große Rolle bei der Qualität und Quantität der gesamten Forschungsaktivität einer Einrichtung $(1,3)$. Folgerichtig ist es von großem Interesse, ob Promotionsarbeiten in der Medizin ausreichend unterstützt werden und welche Faktoren die Qualität studentischer Forschung verbessern können.

Bis dato existieren in Deutschland nur wenige Untersuchungen zum Meinungsbild von erfolgreichen Promovenden $(6,10,13)$. Eine dieser Arbeiten (13) wurde kürzlich im Nachhinein den Ergebnissen einer Befragung nicht-promovierter Vertragsärzte (14) gegenüber gestellt (15). Es gibt somit bisher keine Studie, die erfolgreiche und erfolglose Promotionsvorhaben an einer Fakultät miteinander verglichen hat. Diese Herangehensweise wurde in der Arbeit von Kock und Mitarbeitern (6) und in einem Leserbrief (12) zur Publikation von Pabst et al. (10) gefordert. Ebendieser Fragestellung widmet sich die vorliegende Studie.

\section{Teilnehmer und Methodik}

Unter der Prämisse, eine Querschnittserhebung durchzuführen, wurden alle Medizinstudenten des 11. bis 14. Fachsemesters der Charité ( $n=1036$; 560 Frauen [54,1\%] und 476 Männer [45,9\%]) im Januar 2001 angeschrieben. Dies geschah mit der Intention, sowohl Informationen von erfolgreichen als auch von erfolglosen Promovenden zu erhalten. Dadurch war es möglich, der Frage: „Wie unterscheiden sich erfolgreiche von abgebrochenen Dissertationen?“ nachzugehen. Es wurde ein „Bogen zur Promotionssituation" in dreifacher Ausfertigung versandt. Die Fragen folgten chronologisch dem Ablauf eines Promotionsvorhabens, so dass eine Verwendung des Bogens für die Beschreibung eines Abbruchs ebenfalls möglich war. Folglich konnten die befragten Studenten ihre momentane Promotion und/oder eine bzw. zwei abgebrochene Promotionen beschreiben. Die Fragen waren Themenkomplexen wie Betreuungsqualität, Herangehensweise und Ablauf der Promotion sowie ihrem Einfluss auf die Studiendauer zugeordnet. Außerdem wurden handschriftliche Verbesserungsvorschläge der Befragten erfasst. Bei abgebrochenen Promotionen wurden Abbruchgründe erfragt. Fragen zu rechtlichen und organisatorischen Regelungen an der Charité, zur Qualität der Vorbereitung durch das Studium und zu einem Promotionsvertrag waren in diesem, aber auch in einem zweiten Fragebogen für alle Studenten ohne bisherigen Promotionsversuch („Keine Promotion“) enthalten. Mit Hilfe dieses Bogens wurden Gründe für den Nichtbeginn einer Promotion identifiziert.

Für den Vergleich des Erfolgs eines Promotionsvorhabens wurden zwei Gruppen definiert: einerseits eine Gruppe von Studenten, die ihre Promotionsarbeit eingereicht hatten („Erfolgreiche“) und andererseits eine Gruppe, die ihr Promotionsvorhaben abbrachen („Abbrecher“). Personen, auf die beides zutraf, wurden, um einen intrapersonellen Vergleich zu vermeiden, keiner der beiden Gruppen zugeordnet.

Die statistische Auswertung erfolgte mit dem Programm SPSS 9.0 für Windows. Grundsätzlich wurden zur Analyse hierbei Methoden der deskriptiven Statistik verwandt. Für die Untersuchung der Vergleichsgruppen wurde der $\chi^{2}$-Test für Vierfeldertafeln angewendet. Da die Gruppen der erfolgreichen und abgebrochenen Vorhaben auf Unterschiede bei 12 Parametern untersucht wurden, erfolgte eine Niveau-Adjustierung zur Reduktion des Fehlers 1. Art. Ein $\mathrm{p}<0,0042$ wurde folgerichtig als signifikant definiert. Die Werte sind (wenn nicht anders bezeichnet) als Mittelwert \pm Standardabweichung angegeben.

\section{Ergebnisse}

203 Frauen (55,5\%) und 147 Männer (40,2\%) nahmen an der Umfrage teil. Bei 16 Personen fand sich keine Geschlechtsangabe. Insgesamt beteiligten sich somit 366 Studenten an der Umfrage. Dies entsprach einem Rücklauf von 35\%. Getrennt nach Geschlechtern ergab sich ein Rücklauf von 36\% bzw. 30\% für Frauen und Männer. Der Altersmittelwert in der Umfrage betrug 27,2 \pm 2,5 Jahre. 88\% aller teilnehmenden Personen waren zwischen 25 und 30 Jahren alt. Die Maximalwerte betrugen 23 und 42 Jahre.

\section{Fragebogen zur Promotionssituation}

Insgesamt gingen 382 Bögen von 324 Studenten (31\% Rücklaufquote, 180 Frauen und 132 Männer, 12 ohne Angabe) ein. Es wurden 34 Promotionsvorhaben an anderen Universitäten und Lehrkrankenhäusern der Charité beschrieben. Diese wurden für die weitere Auswertung nicht berücksichtigt. Von den somit verbliebenen 348 Bögen (von 297 Studenten) bezogen sich 271 auf die momentane und 61 bzw. 10 Umfragebögen auf die erste bzw. zweite abgebrochene Promotion. Sechs der Bögen waren durch die Befragten nicht eindeutig in diese Kategorien eingeordnet. Daraus ergibt sich ein Verhältnis von 71 abgebrochenen zu 271 momentan bearbeiteten Promotionen. Folglich werden mindestens $21 \%$ (71 von 342) aller Promotionsvorhaben an der Charité abgebrochen, da davon auszugehen ist, dass von den momentanen Promotionen noch weitere abgebrochen werden.

Das Verhältnis von Frauen und Männern innerhalb der Gruppe der Promotionen an der Charité betrug 164 (55\%) zu 123 (43\%). 10 Personen (3\%) machten keine Geschlechtsangabe. Von den insgesamt 73 wissenschaftlichen Einrichtungen der Charité waren 65 mit mindestens einer beschriebenen Promotion vertreten (89\%). Es fehlte keine der großen vorklinischen oder klinischen Einrichtungen. Die Häufigkeit der verschiedenen Wege zum Promotionsangebot gibt Abb.1 wieder.

\section{Vergleichsgruppen}

Die Unterschiede zwischen den erfolgreichen und abgebrochenen Arbeiten zeigt die Tab.1.

Nach den definierten Kriterien wurden 72 bzw. 65 Arbeiten in die Gruppe der erfolgreichen bzw. abgebrochenen Promotionsvorhaben eingeordnet. In der Gruppe der Erfolgreichen befanden sich 30 Frauen (42\%) und 38 Männer (53\%), bei vier Personen 


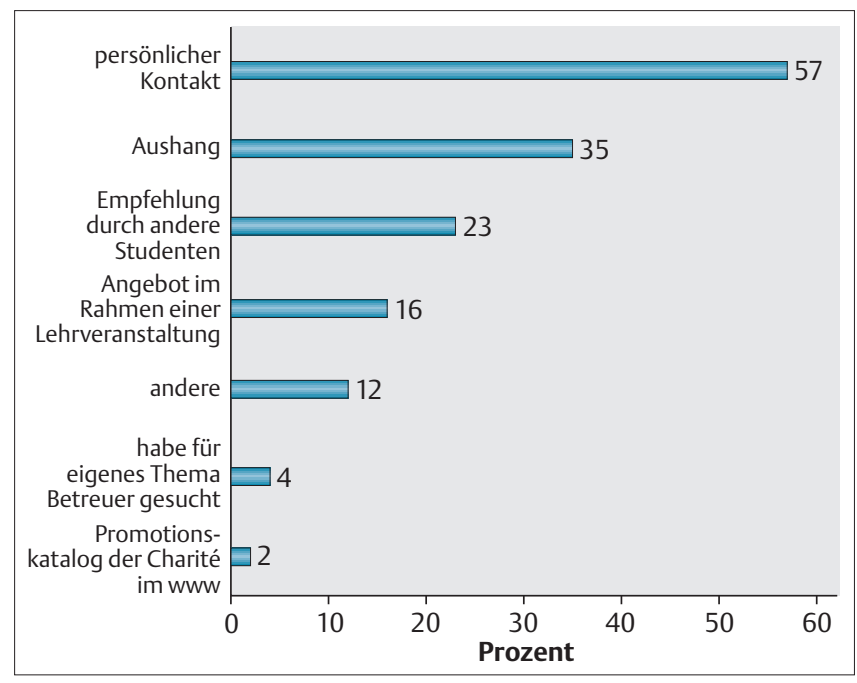

Abb.1 Informationsmöglichkeiten zu Promotionsangeboten und Häufigkeit ihrer Nutzung (Mehrfachantworten möglich, $n=297$ ).

Tab.1 Vergleich der Beurteilung der Promotionssituation in der Gruppe der erfolgreichen und abgebrochenen Vorhaben.

\begin{tabular}{|c|c|c|c|}
\hline & $\begin{array}{l}\text { Erfolgreiche } \\
(n=72)\end{array}$ & $\begin{array}{l}\text { Abbrecher } \\
(n=65)\end{array}$ & \\
\hline $\begin{array}{l}\text { Thematische Einarbeitung } \\
\text { vorhanden }\end{array}$ & $99 \%$ & $97 \%$ & n.s. \\
\hline $\begin{array}{l}\text { Hilfe bei der Einarbeitung } \\
\text { vorhanden }\end{array}$ & $93 \%$ & $83 \%$ & n.s. \\
\hline $\begin{array}{l}\text { Gute Information über die } \\
\text { Thematik }\end{array}$ & $59 \%$ & $32 \%$ & $p=0,002$ \\
\hline Klar formulierte Fragestellung & $60 \%$ & $42 \%$ & n.s. \\
\hline $\begin{array}{l}\text { Vorhandensein eines } \\
\text { Ansprechpartners }\end{array}$ & $99 \%$ & $94 \%$ & n.s. \\
\hline Betreuer mit ausreichend Zeit & $64 \%$ & $29 \%$ & $p<0,001$ \\
\hline $\begin{array}{l}\text { Zusätzlicher Promotions- } \\
\text { berater vorhanden }\end{array}$ & $22 \%$ & $6 \%$ & n.s. \\
\hline Es gab einen klaren Zeitplan & $36 \%$ & $20 \%$ & n.s. \\
\hline $\begin{array}{l}\text { Zusagen über den Umfang } \\
\text { eingehalten }\end{array}$ & $42 \%$ & $5 \%$ & $p<0,001$ \\
\hline $\begin{array}{l}\text { Finanzielle Unterstützung } \\
\text { vorhanden }\end{array}$ & $39 \%$ & $8 \%$ & $\mathrm{p}<0,001$ \\
\hline $\begin{array}{l}\text { Gute Hilfestellung bei akuten } \\
\text { Problemen }\end{array}$ & $57 \%$ & $9 \%$ & $\mathrm{p}<0,001$ \\
\hline $\begin{array}{l}\text { Bemühen, den Studenten als } \\
\text { zukünftigen Forschungs- } \\
\text { partner zu gewinnen }\end{array}$ & $50 \%$ & $17 \%$ & $p<0,001$ \\
\hline n.s. $=$ nicht signifikant & & & \\
\hline
\end{tabular}

fand sich keine Geschlechtsangabe. Zur Gruppe der Abbrecher gehörten 36 Frauen (57\%) und 26 Männer (38\%). Bei drei Personen fehlte die Geschlechtsangabe. Der $\chi^{2}$-Test auf die Geschlechtsverteilung in den beiden Erfolgsgruppen ergab ein $p=0,112$. Der Altersmittelwert in den Gruppen betrug 27,3 $\pm 2,4$ bzw. 27,0 \pm 2,3 Jahre. In der Gruppe der eingereichten Arbeiten ergab die Untersuchung der Dauer, dass im Mittel 27,0 \pm 16,1 Monate bis zur Vollendung der gesamten Dissertation benötigt wurden. 12 (17\%) der erfolgreichen Arbeiten wurden ohne Semesterverlust durchgeführt, 24 (33\%) bzw. 26 (36\%) verlängerten das Studium um ein bzw. zwei Semester und 4 (6\%) Personen benötigten mehr als zwei Freisemester für die Promotion. 6-
Tab.2 Gründe für den Abbruch einer Promotion (Mehrfachantworten möglich, $n=65$ ).

\begin{tabular}{ll}
\hline & Abbruchgründe \\
\hline betreuerische & $86 \%$ \\
methodische & $68 \%$ \\
thematische & $52 \%$ \\
persönliche & $32 \%$ \\
zeitliche & $31 \%$ \\
andere & $25 \%$ \\
finanzielle & $14 \%$ \\
\hline
\end{tabular}

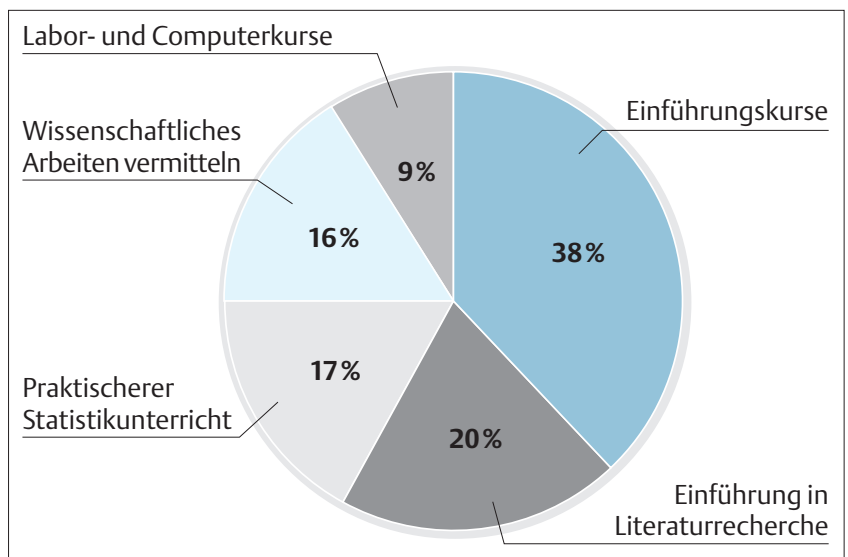

Abb.2 Verbesserungsvorschläge zur derzeitigen Promotionssituation $(n=270)$.

mal erfolgte hierzu keine Angabe. Innerhalb der Gruppe der Abbrecher wurden die Abbruchgründe untersucht. Eine Übersicht zeigt Tab.2.

\section{Verbesserungsvorschläge}

219 (78\%) Personen äußerten sich im „Fragebogen zur Promotionssituation" in freier Textform zu wünschenswerten Verbesserungen der momentanen Situation. Insgesamt wurden 396 Vorschläge genannt. Die häufigsten unter ihnen (jeweils>20-mal) sind in Abb.2 wiedergegeben. Weitere Verbesserungsvorschläge waren (Anzahl der Nennungen in Klammern): zentrale Beratungsstelle (16), Einbindung aktueller Forschungsergebnisse in die Pflichtlehre (14), Einhaltung der vereinbarten Forschungsdauer (14), bessere Betreuung (14) und finanzielle Förderung der Doktoranden (13), kontinuierliche Evaluation der Betreuungsqualität (12), stärkerer Austausch zwischen Doktoranden (7), zentrale obligate Angebotsliste (7), automatische Verleihung des Doktortitels mit Ende des Studiums (4). Außerdem wurden genannt: geringere Arbeitszeitbelastung für Betreuer, höherer Wert einer betreuten Promotion für den Karriereweg des Betreuers, englische Veranstaltungen, Journalclubs, klarere Fragestellungen, flexibleres Studium, mehr Ehrlichkeit und Respekt, sowie eine Schulung der Betreuer (insgesamt 25).

\section{Fragebogen „Keine Promotion“}

Es beteiligten sich insgesamt 42 Personen an der Beantwortung dieses Bogens (23 Frauen und 15 Männer). Auf vier Bögen gab es keine Geschlechtsangabe. Der Altersmittelwert betrug 28,3 $\pm 3,3$ Jahre. 27 (64\%) dieser Personen hatten schon einen potenziellen 
Tab.3 Gründe für den Nichtbeginn einer Promotion (Mehrfachantworten möglich, $n=27$ ).

\begin{tabular}{|ll}
\hline & Gründe für den Nichtbeginn \\
\hline betreuerische & $41 \%$ \\
methodische & $33 \%$ \\
thematische & $41 \%$ \\
persönliche & $56 \%$ \\
zeitliche & $78 \%$ \\
andere & $22 \%$ \\
finanzielle & $41 \%$ \\
\hline
\end{tabular}

Betreuer kontaktiert. Die Gründe für diese Personen vom Beginn einer Promotion Abstand zu nehmen zeigt Tab.3.

\section{Generelle Fragen}

Die folgenden Fragen wurden in beide Fragebogen integriert, so dass sowohl von Promovenden an der Charité (297) als auch von Studenten ohne bisherige Promotion (42) Bewertungen vorliegen. Gefragt nach der Kenntnis der „Grundsätze der Charité zur Sicherung guter wissenschaftlicher Praxis" antworteten 30 (9\%) mit „ja“, 307 (91\%) mit „nein“. Zwei Personen machten hierzu keine Angaben. Die Teilmenge der Studenten ohne Promotion zeigte bei dieser Frage keine deutlichen Abweichungen von der Gesamtheit. Die Promotionsordnung der Charité kannten 167 (49\%), 83 (25\%) nicht, 86 (25\%) hatten von ihr gehört. Drei Personen machten hierzu keine Angaben. Innerhalb der Gruppe der Studenten ohne Promotion war die Promotionsordnung nur 6 (14\%) Personen bekannt. Den www-Promotionskatalog der Charité (http://www.charite.de/fakultaet/ promo_habil/Promotion/promkatalog.html) kannten 84 (25\%), 197 (58\%) nicht, 55 (16\%) hatten davon gehört. Drei Personen machten hierzu keine Angaben. In der Gruppe ohne Promotion war der Promotionskatalog mit 15 (36\%) etwas besser bekannt. Die Antworten auf die Frage, wie das Studium auf wissenschaftliches Arbeiten vorbereitet, sind in Abb. $\mathbf{3}$ dargestellt. Es fand sich hierbei kein deutlicher Unterschied zwischen beiden Gruppen. Gefragt nach der Zustimmung zu einem Promotionsvertrag, der sowohl die Verpflichtungen von Promovend und Betreuer als auch die Fragestellung und Rahmenbedingungen der Arbeit festlegen sollte, antworteten 258 (76\%) positiv. Ablehnend zeigten sich 29 (9\%), und 43 (13\%) konnten sich nicht festlegen. Neunmal wurde keine Angabe gemacht. Auch bei dieser Frage gab es keine deutlichen Differenzen zwischen den beiden Personengruppen.

\section{Diskussion}

\section{Rücklauf}

324 (31\%) der Befragten sandten mindestens einen „Fragebogen zur Promotionssituation“ zurück. Auf den ersten Blick erscheint dies wenig. Unter der Maßgabe, dass die meisten Promotionsvorhaben innerhalb von 5 Jahren abgeschlossen werden $(6,10,13)$, sollten folgende Zahlen jedoch berücksichtigt werden:

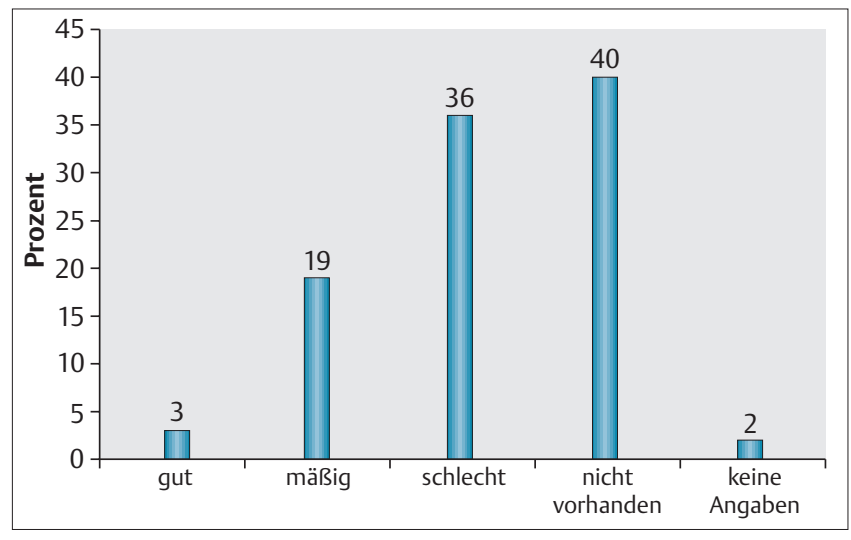

Abb.3 Qualität der Vorbereitung auf wissenschaftliches Arbeiten durch das Studium $(\mathrm{n}=339)$.

- Nur 38\% aller Studenten haben 5 Jahre nach dem Studium eine Promotion abgeschlossen (8);

rund 36\% aller in Berlin tätigen Ärzte unter 35 Jahren sind promoviert (Mitteilung der EDV-Abteilung der Berliner Ärztekammer).

Da 31\% der Studenten ihr Promotionsvorhaben beschrieben, ist somit von einem Rücklauf innerhalb der betrachteten Gruppe von über $50 \%$ und damit von einer repräsentativen Umfrage auszugehen. 89\% aller wissenschaftlichen Einrichtungen der Charité sind durch die Befragung erfasst worden. 42 (4,1\%) Studenten antworteten auf den Fragebogen „Keine Promotion“. Die Ergebnisse innerhalb dieser Untergruppe sind nicht als repräsentativ zu werten. Anscheinend ist es nicht in ausreichendem Maße gelungen, die Wichtigkeit auch dieses Teils der Umfrage zu vermitteln.

Insgesamt sind derzeit in Berlin 58\% aller tätigen Ärzte promoviert (Mitteilung der EDV-Abteilung der Berliner Ärztekammer). Eine bundesweite Schätzung aufgrund des Verhältnisses der Promotionen zu den Absolventen in der Medizin in Deutschland ergab hingegen eine Steigerung von 59\% im Jahr 1985 auf 74\% im Jahr 1999 (9). Diese Berechnung vernachlässigt jedoch den Rückgang der Absolventen in den 90 -er Jahren um $20 \%$ (4), der diese scheinbare Steigerung der promovierten Ärzte bewirken könnte. Eine Studie zur Feststellung des tatsächlichen bundesweiten Anteils der promovierten Ärzte sollte folgerichtig zur Klärung durchgeführt werden.

\section{Ergebnisse}

Da in Deutschland mit Einführung der Juniorprofessur die Habilitation abgelöst werden soll (11), ist es sehr wahrscheinlich, dass die Qualität der Promotion zur zukünftigen Messlatte für eine wissenschaftliche Karriere werden wird. Gerade auch für den klinisch tätigen Mediziner ist in Anbetracht von „Evidencebased Medicine“ (2) eine Beschäftigung mit wissenschaftlichen Fragestellungen, z.B. im Rahmen einer Promotion, von großer Bedeutung. Deshalb liegt ein besonderes Augenmerk auf der Frage, wie sich erfolgreiche von abgebrochenen Promotionen unterscheiden, damit nachgerade aus diesen Ergebnissen Ansatzpunkte für eine Qualitätsverbesserung abgeleitet werden können. 
Die erfolgreichen Promotionsvorhaben zeigten deutliche Differenzen im Vergleich zu den abgebrochenen Vorhaben. Diese betreffen 1) betreuerische Aspekte und 2) strukturelle Gesichtspunkte. Sehr illustrativ war in diesem Zusammenhang auch die subjektive Einschätzung des Bemühens um den Studenten als zukünftigen Forschungspartner. Es ist des Weiteren davon auszugehen, dass mindestens jedes fünfte Promotionsvorhaben abgebrochen wird. Die maßgeblichen Gründe für einen Abbruch sind betreuerischer (86\%) und methodischer (68\%) Natur. Somit sind für den Erfolg einer Promotion vor allem eine gute Supervision durch den Betreuer und eine klare Planung und Strukturierung der Arbeit wichtig. Dies wird besonders deshalb verständlich, da die Studenten durch das Studium zu über 75\% gar nicht oder schlecht auf die Promotion vorbereitet werden. Keine Verlängerung des Studiums wurde bei einem Sechstel der erfolgreichen Promotionen beobachtet. Jeweils rund ein Drittel der erfolgreichen Arbeiten bewirkte eine ein- bzw. zweisemestrige Verzögerung des Studiums und 6\% kosteten mehr als zwei Semester. Diese Ergebnisse zeigen einen stärkeren Einfluss der Promotion auf die Studiendauer als in bisherigen Untersuchungen (keine Studienverlängerung bei 44\% [6], 52\% [10] bzw. 66\% [13]). Eine stärkere Integration der Promotion ins Studium und dadurch gewährleistete bessere Vorbereitung könnte eine günstigere zeitliche Effizienz bewirken. In der Gruppe der abgebrochenen Promotionen sind im Vergleich der Geschlechter tendenziell häufiger Frauen vertreten $(p=0,112)$. Ein derartiger Unterschied zu Ungunsten der Frauen ist somit denkbar, jedoch nicht nachgewiesen.

Besonderer Nachholbedarf bei der Vorbereitung auf die Promotion wurde vor allem in den Teilbereichen: Statistik, Literaturrecherche und Arbeit mit dem Computer und im Labor gesehen. Aber auch ein schriftlicher Promotionsvertrag, der beiderseitige Rechte und Pflichten definiert, traf bei über $75 \%$ auf Zustimmung. Die Tatsache, dass die Promotionsordnung und noch stärker die Grundsätze zur Sicherung guter wissenschaftlicher Praxis nur zu 49 bzw. 9\% allen Befragten bekannt waren, gibt entsprechend Anlass zur Sorge. Auf die Vermittlung von derartigen rechtlichen Grundlagen und Grundsätzen für das tägliche Handeln wird anscheinend nicht ausreichend geachtet. Der www-Promotionskatalog wurde bisher nur einem Viertel der Studenten als Medium vermittelt. Folglich spielt diese ideale Kommunikationsmöglichkeit auch bei der Suche nach Angeboten kaum eine Rolle, vielmehr sind der persönliche Kontakt und Aushänge die häufigen Wege zu einem Promotionsthema.

\section{Fazit}

Die vorliegende Studie zeigt, dass es vor allem darum gehen muss, die betreuerische und strukturelle Qualität von Promotionsvorhaben zu erhöhen. Dazu sollten folgende Punkte realisiert werden:

- vorbereitende und begleitende Kursangebote zum Einstieg in wissenschaftliches Arbeiten (5),

- stärkere Integration der Promotion in den Studienablauf unter Beibehaltung des derzeitigen Systems der fakultativen studentischen Forschung,
- Promotionsverträge zur genauen Definition der beiderseitigen Verpflichtungen und Festlegung der Zielstellung der Forschungsarbeit,

bessere Vermittlung der rechtlichen und praktischen Grundlagen, z.B. auf dem Weg der Vereinfachung derselben durch Reduktion auf Kernsätze,

- kontinuierliche Evaluation der Betreuung.

Wissenschaftsstandorte und deren Zukunft wird durch die Qualität des Nachwuchses maßgeblich determiniert. Deshalb wird es entscheidend sein, die medizinische Promotion bei gleich bleibender zeitlicher Flexibilität stärker in den Studienablauf zu integrieren. Nur dann kann es gelingen - stärker als bei den konkurrierenden MD/PhD-Modellen - neben der Qualität auch die kritische Masse exzellenter Nachwuchswissenschaftler zu erreichen.

Danksagung: Mein Dank gilt R. Siewczynski und PD C. Dewey für ihre inhaltliche Unterstützung bei der Planung der Untersuchung; A. Ruszczynski, I. Petruschke, A. Lasch, E. Zimmermann und E. Schönenberger für ihre Hilfe bei der Organisation der Studie und den anonymen Gutachtern für ihre hilfreichen Kommentare. Außerdem möchte ich der Ständigen Kommission für Lehre und Studium der Charité für die finanzielle Förderung der Durchführung der Studie danken.

\section{Literatur}

1 Altunbas A, Cursiefen C. Forschungsaktivität von Medizinstudenten in Deutschland am Beispiel der Universitätsklinik Würzburg. Dtsch med Wochenschr 1998; 123: 617-620

2 Antes G, Bassler D. Evidence-Based Medicine, Forschungstransfer und die Rolle der medizinischen Journale. Dtsch med Wochenschr 2000; 125: 1119-1121

3 Chen J. The youth team. Nature 2001; 411: 13-14

4 Clade H. Reform des Medizinstudiums: Auf der Zielgeraden. Dtsch Ärztebl 2001; 98: A2019-2023

5 Dewey M, Petruschke I, Lasch A, Zimmermann E, Schönenberger E. Computerbasierte Ausbildung: Medizinstudenten ergriffen Initiative. Dtsch Ärztebl 2001; 98: A3180-3181

6 Kock N, Gauer IC, Busch LC, Kirchner H. Betreuung medizinischer Doktoranden im interuniversitären Vergleich - Sollte das Promotionsverfahren geändert werden? Dtsch med Wochenschr 2000; 125: 724-728

Mervis J. Student Research: What is it good for? Science 2001; 293: 16141615

8 Minks KH, Bathke G. Absolventenreport Medizin - Ergebnisse einer Längsschnittuntersuchung zum Berufsübergang von Absolventinnen und Absolventen der Humanmedizin. Bundesministerium für Bildung und Wissenschaft, Referat Öffentlichkeitsarbeit, Bonn, 1994

9 Pabst R. Aktuelle Herausforderungen in der Hochschulmedizin. Die Humanmedizin im Portrait. Forschung und Lehre 2001; 6: 309-312

10 Pabst R, Strate J, Rothkötter HJ. Die medizinische Dissertation: Sinnvolle Ergänzung - oder Ablenkung vom Studium? Dtsch Ärztebl 1997; 94: A23142318

11 Richter E. Hochschulen: Reform für mehr Wettbewerb. Dtsch Ärztebl 2001; 98: A3252

12 Schwahn J. Dissertation: Kontrollgruppe fehlt. Dtsch Ärztebl 1998; 95: A116

13 Weihrauch M, Weber A, Weltle D, Pabst R, Lehnert G. Der Weg zum Dr. med. - wie beurteilen Doktoranden ihre Dissertation? Dtsch med Wochenschr 1998; 123: 375-380

14 Weihrauch M, Weber A, Weltle D, Lehnert G. „Doktor“ ohne Dr. med. - wie beurteilen nicht-promovierte Vertragsärzte medizinische Dissertationen? Gesundheitswesen 1999; 61: 255-259

15 Weihrauch M, Weber A, Pabst R, Weltle D, Lehnert G. Die medizinsche Dissertation: Eine Bestandsaufnahme aus der Sicht erfolgreicher und gescheiterter Promovenden. Med Klin 2000; 95: 545-547 